\title{
Compound Kushen Injection
}

National Cancer Institute

\section{Source}

National Cancer Institute. Compound Kushen Injection. NCI Thesaurus. Code C120314.

A traditional Chinese medicine ( $\mathrm{TCM}$ ) formulation composed of compound Kushen injection (CKI) containing aqueous extracts from the roots of Kushen (Radix Sophorae Flavescentis) and Baituling (Rhizoma smilacis Glabrae), with potential antineoplastic and immunomodulating activities. CKI contains numerous chemicals including alkaloids, such as matrine and oxymatrine, flavonoids, alkylxanthones, quinones, triterpene glycosides, fatty acids, and essential oils. Although the exact mechanism(s) of action through which CKI exerts its effects has yet to be fully elucidated, CKI is able to interfere with the activation of various signal transduction pathways, such as the Wnt/beta-catenin signaling pathway, inhibit nuclear factor-kappa B (NF-KB) activation, and block the activity of multiple receptor tyrosine kinases, such as epidermal growth factor receptor (EGFR) and vascular endothelial growth factor receptor (VEGFR). CKI induces apoptosis in and inhibits proliferation, migration, invasion and adhesion of tumor cells. CKI also modulates the production of inflammatory mediators. 